\title{
Activation of Cerebral X-linked Adrenoleukodystrophy After Head Trauma
}

\author{
Adrian Budhram, Sachin K. Pandey
}

Keywords: Leukodystrophy, head trauma

doi:10.1017/cjn.2017.52

Can J Neurol Sci. 2017; 44: 597-598

A 21-year-old man with X-linked adrenoleukodystrophy (X-ALD) suffered a traumatic brain injury after a motor vehicle accident. He had been diagnosed with X-ALD after developing adrenal insufficiency ten years prior, but had no known neurologic involvement. Head magnetic resonance imaging (MRI) at the time of his motor vehicle accident revealed bilateral, frontalpredominant cerebral microbleeds, with no white matter abnormalities (Figure 1). After a 1-month hospital admission, he was transferred to rehabilitation, and during 2 months there, he reattained independence of activities of daily living. Unfortunately, he then suffered an acute neurocognitive decline, with inattention, disorientation, apathy, and impaired memory. Motor, sensory, and coordination testing was otherwise grossly unremarkable. Repeat gadolinium-enhanced MRI of the head showed interval

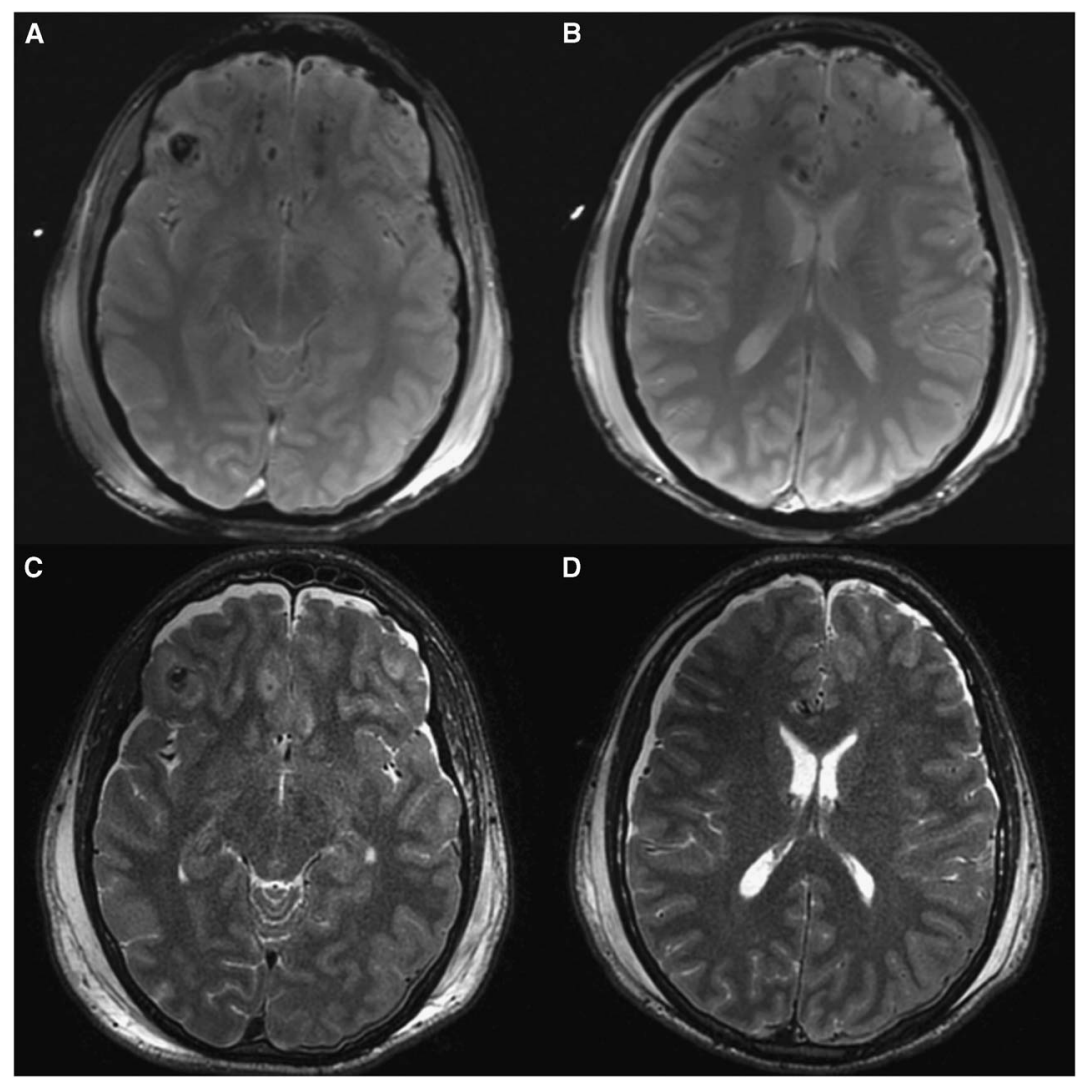

Figure 1: Magnetic resonance imaging of the head at time of head injury. Axial MPGR images $(A, B)$ show bilateral, predominantly frontal scattered hypointensities, consistent with traumatic microbleeds. Axial T2-weighted images $(C, D)$ show no evidence of cerebral X-linked adrenoleukodystrophy, with normal-appearing white matter.

From the Departments of Clinical Neurological Sciences (AB) Medical Imaging (SKP), London Health Sciences Centre, London, Ontario, Canada. Received November 10, 2016. Date of AcCePtance DeCember 12, 2016.

Correspondence to: Adrian Budhram, Department of Clinical Neurological Sciences, London Health Sciences Centre, 339 Windermere Rd., London ON, Canada, N6A 5A5.

E-mail: adrian.budhram@medportal.ca 


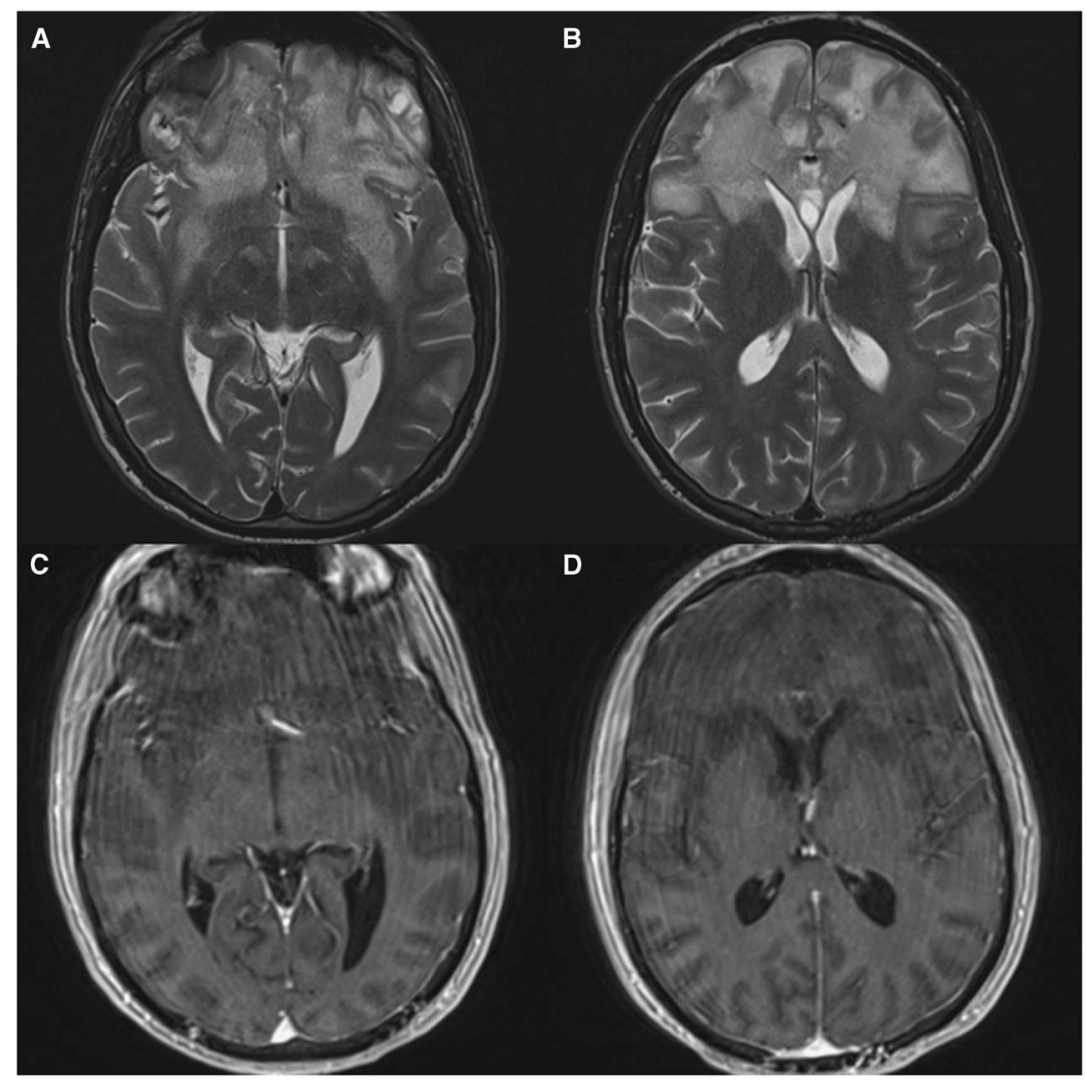

Figure 2: Gadolinium-enhanced magnetic resonance imaging of the head 4 months after head injury. Axial T2-weighted images $(A, B)$ show interval development of bilateral, predominantly frontal, symmetric, confluent white matter hyperintensities, compatible with cerebral X-linked adrenoleukodystrophy (X-ALD) after head trauma. Axial T1-weighted postgadolinium images $(C, D)$ do not show any leading edge enhancement characteristic of cerebral $X-A L D$.

development of symmetric, confluent, frontal-predominant white matter T2-hyperintensities, without enhancement (Figure 2). Given his medical history, he was diagnosed with cerebral activation of X-ALD after head trauma. ${ }^{1,2}$

$\mathrm{X}$-ALD is a disease of the adrenal cortex and nervous system white matter. It is caused by mutation of the $A B C D 1$ gene, which encodes a protein involved in breakdown of very long-chain fatty acids (VLCFAs). ${ }^{1,2}$ Disease phenotypes include a childhood cerebral form, adrenomyeloneuropathy in early adulthood, and adrenal insufficiency. Cerebral X-ALD after head trauma is rare and challenging diagnostically if there is no patient or family history of the disease. The pathophysiology is poorly understood, but may be related to biochemical cascades after trauma that promote inflammation, inhibit VLCFA breakdown, and lead to increased VLCFA incorporation into myelin. ${ }^{1}$ Our patient provides one of the clearest published accounts of cerebral X-ALD triggered by head trauma with no clinical or radiographic evidence of cerebral X-ALD at the time of injury. Three months after his traumatic brain injury, he suffered an acute neurocognitive decline with new, confluent, frontal-predominant white matter abnormalities on MRI, bolstering the plausibility of cerebral X-ALD triggered by frontal-predominant traumatic microbleeds. Importantly, our case highlights how the white matter abnormalities in cerebral X-ALD may be most prominent at the site of cerebral injury, rather than in the parietooccipital pattern that is typically described. The leading-edge enhancement characteristic of cerebral X-ALD was not seen, demonstrating that absence of this finding is not incompatible with diagnosis. The recognition of this rare entity may be instrumental to avoiding excessive and invasive diagnostic testing, such as brain biopsy. A delay may occur between the time of head trauma and clinicoradiographic onset of cerebral X-ALD, suggesting this diagnosis merits consideration even months after head injury. Close monitoring of patients with X-ALD after head injury may be warranted, particularly if disease-modifying therapy becomes available.

\section{Statement of Authorship}

$\mathrm{AB}$ reviewed the clinical history, reviewed and formatted the neuroimaging, and drafted the manuscript. SKP reviewed the neuroimaging, and edited the drafted manuscript for its intellectual content.

\section{DisClosures}

The authors have nothing to disclose.

\section{REFERENCES}

1. Raymond GV, Seidman R, Monteith TS, et al. Head trauma can initiate the onset of adreno-leukodystrophy. J Neurol Sci. 2010;290:70-4.

2. Bouquet F, Dehais C, Sanson M, Lubetzki C, Louapre C. Dramatic worsening of adult-onset X-linked adrenoleukodystrophy after head trauma. Neurology. 2015;85:1991-3. 